\title{
Universiteit
}

Leiden

The Netherlands

\section{Cognitive load dissociates explicit and implicit measures of body ownership and agency}

Qu, J.; Ma, K.; Hommel, B.

\section{Citation}

Qu, J., Ma, K., \& Hommel, B. (2021). Cognitive load dissociates explicit and implicit measures of body ownership and agency. Psychonomic Bulletin \& Review, 28, 1567-1578. doi:10.3758/s13423-021-01931-y

Version:

Publisher's Version

License:

Licensed under Article 25fa Copyright Act/Law (Amendment Taverne)

Downloaded from: https://hdl.handle.net/1887/3248697

Note: To cite this publication please use the final published version (if applicable). 


\title{
Cognitive load dissociates explicit and implicit measures of body ownership and agency
}

\author{
Jue $\mathrm{Qu}^{1} \cdot \mathrm{Ke} \mathrm{Ma}^{1}$ (D) $\cdot$ Bernhard Hommel $^{2,3}$ \\ Accepted: 7 April 2021 / Published online: 25 May 2021 \\ (C) The Psychonomic Society, Inc. 2021
}

\begin{abstract}
It is often claimed that the human self consists of perceived body ownership and agency, which are commonly assessed through explicit ownership and agency judgments and implicit measures, like proprioceptive drift, skin conductance responses, and intentional binding effects. Bottom-up multisensory integration and top-down modulation were predicted to be important for ownership and agency. In previous studies, cognitive load was revealed to affect the sense of agency in a top-down fashion, but its effect on ownership has not been fully investigated, not even its possibly different effect on explicit and implicit measures. Here we used cognitive load (small vs. large sets in a working-memory task) to disentangle explicit and implicit measures of ownership and agency in a task inducing the virtual hand illusion (VHI; stronger perceived ownership and agency over a virtual hand if it moves in synchrony with one's real hand). Results showed similar patterns for ownership and agency - both ownership and agency were affected by cognitive load, and importantly in the explicit measures, higher load increased the effect of synchrony (i.e., the VHI), but in implicit measures, higher load reduced indications of both ownership and agency. Hence, the load manipulation was selective with regard to the explicit versus implicit nature of the measure but not with respect to the measure's content. This provides strong evidence that explicit and implicit measures of both ownership and agency rely on at least partly separable informational sources, while the difference between ownership and agency as such does not seem to play a major role.
\end{abstract}

Keywords Sense of ownership $\cdot$ Sense of agency $\cdot$ Cognitive load $\cdot$ Virtual hand illusion $\cdot$ Intentional binding $\cdot$ Proprioceptive drift

\section{Introduction}

Perceived body ownership and agency are assumed to be two important ingredients of the "minimal" self, the experience that we are individuals who differ from others (Blanke \& Metzinger, 2009). More specifically, perceived ownership refers to the experience that we have a body that

$\mathrm{Ke} \mathrm{Ma}$

psyke1@swu.edu.cn

$\triangle$ Bernhard Hommel

bh@bhommel.onmicrosoft.com

1 Key Laboratory of Personality and Cognition, Faculty of Psychological Science, Southwest University, Beibei, Chongqing, China

2 Institute for Psychological Research and Leiden Institute for Brain and Cognition, Leiden University, Leiden, The Netherlands

3 Department of Psychology, Shandong Normal University, Jinan, China belongs to us, while agency refers to the experience of being the cause or author of an action (Gallagher, 2000, 2007; Moore \& Fletcher, 2012). Ownership and agency have often been investigated by means of the virtual hand illusion (VHI; Slater et al., 2008; Sanchez-Vives et al., 2010), a virtual version of the rubber hand illusion (RHI; Botvinick \& Cohen, 1998; Pfister et al., 2020), in which participants are confronted with virtual extensions of their body on a screen or presented through virtual reality head-mounted displays, while wearing a data glove that translates movement of their real hand into movements of the virtual effector. When the real and virtual hands move in synchrony, participants have a much stronger feeling that the virtual effector belongs to them (sense of ownership) and that they can cause or control the action of this virtual effector (sense of agency) than when the real and virtual hands move asynchronously (e.g., with a temporal delay of the former to the latter). To assess these effects, researchers have used explicit measures, like questionnaires on which participants rate the degree of perceived ownership and agency (Botvinick \& 
Cohen, 1998), and implicit measures, that are thought to reflect the non-conscious aspect of ownership and agency, like a proprioceptive drift of one's real effector towards the virtual effector (Tsakiris \& Haggard, 2005) and skin conductance responses (SCR) to apparent threats to the virtual effector (Armel \& Ramachandran, 2003; Ehrsson, 2012) to assess ownership and intentional binding (IB; Haggard et al., 2002) to assess agency.

If explicit and implicit measures of ownership would measure the same psychological function, and if this function would systematically differ from the psychological function that is measured by explicit and implicit measures of agency, one would expect strong correlations between the explicit and implicit measures of ownership and between the explicit and implicit measures of agency, but weak or non-existing correlations between explicit or implicit measures of ownership and explicit or implicit measures of agency. However, increasing evidence suggests a different pattern. For instance, in our own VHI studies (e.g., Ma et al., 2021; Ma \& Hommel, 2015b), we usually found tight correlations between explicit measures of ownership and agency, suggesting that whatever these explicit measures measure overlaps to a considerable degree. One possible reason for these observations might be the fact that the experimental setup generating a VHI is much closer to daily experience of participants than RHI setups, from which dissociations between explicit measures of ownership and agency have been reported (Kalckert \& Ehrsson, 2012). Indeed, in daily life we often experience ownership and agency together, so that a distinction between the two concepts might be too academic to reflect a distinction of functional relevance.

Indeed, researchers have observed that ownership and agency are often equally affected by experimental conditions, and under certain circumstances one can promote the other (Braun et al., 2018; Pyasik et al., 2018; Tsakiris et al., 2007). Importantly, the idea that perceived ownership and agency might rely on overlapping informational sources raises an interesting theoretical possibility: a theoretical framework that allows for the systematic integration of multisensory information into a more coherent scenario of how perceived ownership and agency can be derived. Thus it is possible that theories of agency can be extended to ownership, and vice versa. For example, Ma et al. (2019a) tested the idea that the exclusivity principle proposed in the agency theory of Wegner (2003) would also be relevant cues for ownership. Results show that exclusivity produced significant effects on both explicit ownership and agency: Ratings were higher when participants controlled the virtual effector alone than when sharing control with a partner. The finding extends Wegner's agency theory to explain perceived body ownership, which in turn provides the possibility of an integrative framework for interpreting constraint factors on ownership and agency illusions.
Thus, the first aim of the present study was to test whether a factor known to impact explicit agency - cognitive load - can also be shown to impact explicit ownership in a VHI setup. Several agency studies unrelated to RHI or VHI clearly demonstrated an effect of cognitive load. For example, Kannape et al. (2014) asked participants to walk towards a goal in a virtual environment, and investigated the effects of cognitive load on participants' motor awareness when seen deviations occurred from the veridical walking trajectory. The authors showed that cognitive load impaired motor awareness and reduced individual motor control and conscious perception under trials with high cognitive load levels and moderate deviations. Hon et al. (2013) used working-memory tasks to manipulate high or low cognitive load, assuming that higher load would lead to a stronger reduction of the contribution of conscious operations to the determination or perception of agency. Ratings were used as explicit measures of agency and the IB (the difference between the time the action was perceived to occur and the time the effect of the action was perceived to occur) as implicit measure. The authors found that explicit ratings were moderated by cognitive load, with lower agency ratings being observed in the high load condition - but the IB effect was not affected. In contrast, Howard et al. (2016) used working-memory tasks with high and low load to study the impact on IB, the implicit agency measure, only to find evidence of reduced agency with higher load. While these observations suggest that load does have an effect on agency, it is not quite clear whether it is targeting explicit measures, implicit measures, or both. Indeed, the relationship between explicit and implicit measures is empirically complex, as indicated by observations that they are often affected differently by experimental manipulations and that intercorrelations between them are mostly low for both ownership (Ma et al., 2019a; Ma \& Hommel, 2015b; Pyasik et al., 2018; Pyasik et al., 2020) and agency (Dewey \& Knoblich, 2014; Ebert \& Wegner, 2010; Haggard, 2017), and theoretically opaque, as indicated by the absence of a coherent theory that identifies the different informational sources on which these measures might rely.

A particularly obvious possible distinction between explicit and implicit measures might refer to the theoretical controversy about top-down and bottom-up contributions to perceived ownership. While some authors have considered the possibility of a pure bottom-up account, which might rely on correlations between body-generated sensory feedback provided by the available sensory modalities (e.g., Botvinick \& Cohen, 1998), others have claimed the existence of stable internal body representations that guide the perception of ownership in a top-down fashion (Tsakiris, 2017). Still others have considered the possibility that the perception of ownership might emerge from multiple sources, which might include both bottom-up and top-down contributions (Apps \& Tsakiris, 2014; Ma \& Hommel, 2020, 2015b; Synofzik et al., 2008a, 
2008b). Multiple-source accounts open the possibility that measures differ with respect to the nature of the underlying informational sources, so that some measures may rely more on top-down or on bottom-up information than others, which may explain the different sensitivities to experimental manipulations and the low correlations between measures. For instance, it makes sense to assume that explicit judgments are more strongly affected by top-down expectations and predictions than implicit measures are, so that explicit measures might be more sensitive to experimental manipulations that affect top-down processing. This is indeed the scenario that was suggested by Hon et al. (2013), who found an effect of load on explicit agency but not on implicit agency, as assessed by IB. Unfortunately, this scenario does not quite fit with the findings of Howard et al. (2016), who observed an impact of load on IB. However, this discrepancy might be accounted for by subtle differences of the employed task: Hon et al. (2013) used a time-interval estimation paradigm to calculate IB, while Howard et al. (2016) used a time-interval reproduction paradigm. Arguably, the latter relies more on top-down cognitive control than the former, which could explain why it is affected more strongly by a load manipulation.

These considerations suggest that manipulations of cognitive load may not only be interesting for comparing explicit measures of ownership and agency, but also for dissociating explicit and implicit measures. Given the preliminary indications that explicit and implicit agency measures might be affected differently by load, we were thus interested to test whether this pattern could be replicated and whether it would also be found for explicit and implicit ownership measures. Accordingly, the second aim of the present study was to test whether cognitive load would affect the relationship between explicit and implicit measures of ownership in the same way as it has been found to affect the relationship between explicit and implicit measures of agency. More specifically, we compared a condition with low working-memory load and a condition with high working-memory load, as used in the studies of Hon et al. (2013) and Howard et al. (2016), to test how widely used explicit and implicit measures of perceived ownership and agency are affected by this manipulation. As pointed out, we were particularly interested to see whether cognitive load can affect ownership, and how the manipulation would structure the outcomes of explicit and implicit measures. The classical self $=$ ownership + agency approach (Gallagher, 2000; Jeannerod, 2003) would suggest that both explicit and implicit ownership measures would show comparable load effects while explicit and implicit agency measures would show a different effect pattern. In contrast, recent findings suggest that explicit measures of ownership and agency are often affected similarly by experimental manipulations, which we also expected to hold for the present load manipulation, and there is increasing evidence that explicit and implicit measures differ in their sensitivity to experimental manipulations (Hon et al., 2013; Howard et al., 2016; Ma \& Hommel, 2015a). Along these lines, we also considered the possibility that the impact of cognitive load on explicit measures differs systematically from the impact of load on implicit measures. We manipulated cognitive load in a standard VHI task. As usual in VHI tasks, participants wore a data glove that translated movements of their real right hand into movements of a virtual hand presented through a head-mounted display, either without any detectable delay (the synchrony condition) or with a substantial temporal delay (the asynchrony condition). After experiencing synchrony or asynchrony, participants rated their perceived ownership and agency over the virtual hand in a standard questionnaire, which provided us with the explicit measures, and we also assessed proprioceptive drift, SCR, and IB to generate implicit measures of body ownership and agency.

\section{Method}

\section{Participants}

Forty-two participants (five males; mean age $=19.73$ years, standard deviation $[\mathrm{SD}]=0.79$, range $18-21$ ) were recruited from Southwest University, China, in exchange for pay. All had normal or corrected to-normal vision, were right-handed, and were naive with respect to RHI/VHI and the tested hypotheses. The study was approved by the local human research ethics committee, written informed consent was obtained from all participants before the experiment, and the methods were carried out in accordance with the approved guidelines. Sample size was determined with reference to a previous agency study with comparable methods (Howard et al., 2016), in which 36 participants were tested. Results of post hoc power analyses are presented below.

\section{Setup}

The experiment adopted the basic VHI setup of Ma and Hommel (2015b). We used the software Vizard to build a virtual reality environment: a right-hand data glove (Manus, 12 sensors, record frequency $200 \mathrm{HZ}$, latency around $5 \mathrm{~ms}$ ) that participants wore on their right hand to record their hand and finger joint movements, an HTC vive tracker participants wore on their wrist to record their hand rotation, and a HTC vive Virtual Reality head-mounted display (HMD) participants wore on their head, through which the virtual hand and other objects were presented (see Fig. 1). We built a three-dimensional (3D) virtual hand model and imported it into the virtual reality environment, and then wrote a script using Vizard, through which the real hand-movement data 


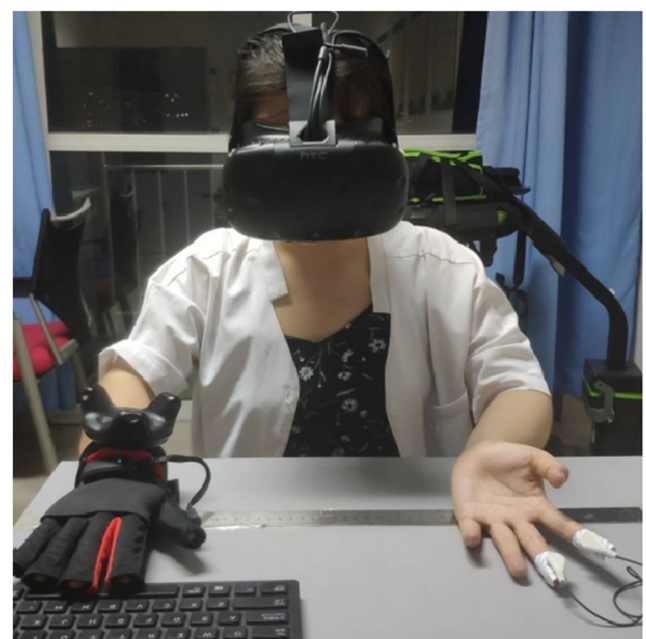

Fig. 1 Experimental setup. (Left panel) Participants wore a headmounted display on their head, and an orientation tracker and dataglove on their right hand. Skin conductance response electrodes were attached to the index and middle fingers of the left hand, and the keyboard was

collected by the data glove sensors could be translated into the $3 \mathrm{D}$ virtual hand. In the synchronous condition, the movement data were directly fed into the movements of the virtual hand, without any detectable delay. In the asynchronous condition, the movement of the virtual hand was delayed by $3 \mathrm{~s}$.

\section{Design}

To determine how cognitive load modulates ownership and agency, we manipulated two independent factors within participants. The first one (load) was manipulated by requiring participants to complete two different memory tasks (Hon et al., 2013; Howard et al., 2016; Sternberg, 1966), which both consisted of a presentation stage and a recall stage in each trial. Between the two stages, participants were exposed to the virtual hand and carried out one IB trial, while keeping the to-bememorized letters in memory until the recall stage. During the presentation phase, two (low load) or eight (high load) randomly selected letters were presented successively for $1,000 \mathrm{~ms}$ each. After exposure to the virtual hand and the IB trial, the recall phase started. A probe letter was shown and participants were to report whether or not the probe letter was presented in the presentation phase. The probability of either the two- or eight-letter list option was set to be $50 \%$ in all trials. The second factor was synchrony, that is, whether the movement of the virtual hand was or was not synchronous with that of the real hand.

As dependent variables we recorded explicit judgments of ownership and agency (Ma \& Hommel, 2015b) and three implicit measures: proprioceptive drift (Tsakiris \& Haggard, 2005) and SCR (Ma \& Hommel, 2013, 2015a) to assess ownership, and IB (Haggard et al., 2002; Ruess et al., 2018) to assess agency.

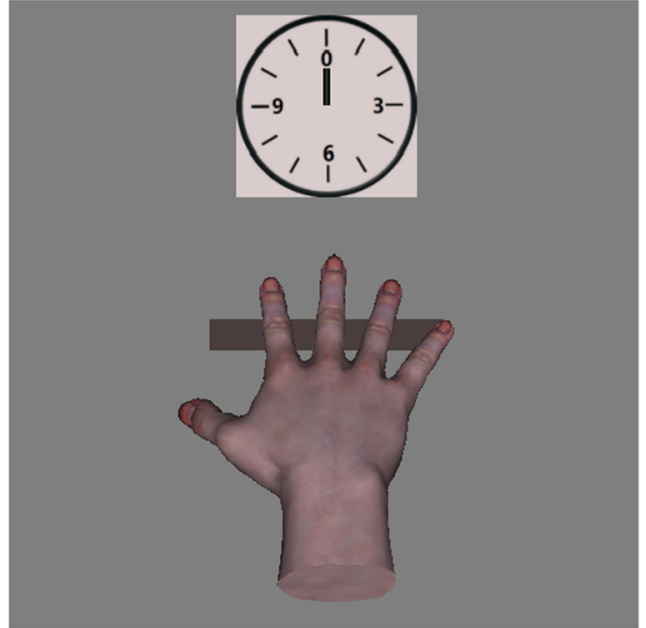

placed before the real hand. (Right panel) The viewed virtual hand and clock for the intentional binding task in the virtual environment carried out by participants

Participants engaged in all four conditions, which resulted from crossing the two load conditions with the two synchrony (synchronous and asynchronous) conditions. The sequence of the four conditions was fully counterbalanced across participants.

\section{Procedure}

The schematic overview of one specific condition example is shown in Fig. 2. Upon arrival in the lab, participants were to put the HMD on their head, the data glove on their right hand, the orientation tracker on their right wrist, and the SCR electrodes on the index and middle fingers of their left hand, which was placed in a relaxed state on the table during the whole experiment. Before the actual experiment started, participants were asked to complete the IB baseline test (Ma et al., 2019b). A virtual clock, its pointer and button (but no virtual hand) were shown in the virtual environment, the original pointer position was set to be at zero, participants were asked to voluntarily press the space key at their will with their real finger on the real keyboard put in front of their real hand. When the real space key was pressed, the virtual button went down and back up, and the virtual clock pointer started to rotate. The clock pointer always rotated with a round of $1,200 \mathrm{~ms}$ for each press, from zero to zero; at a random time point of between 600 and $1,000 \mathrm{~ms}$ after the button was pressed, the script generated a tone.

Participants were to pay attention to the tone and pointer rotation, and report the pointer position at tone occurrence. This baseline test contained 10 IB trials. After the baseline test, participants completed the four experimental conditions.

There were four phases in each of the four conditions. First, the virtual clock and its pointer and button disappeared, and a virtual right hand and a number array under the virtual hand 

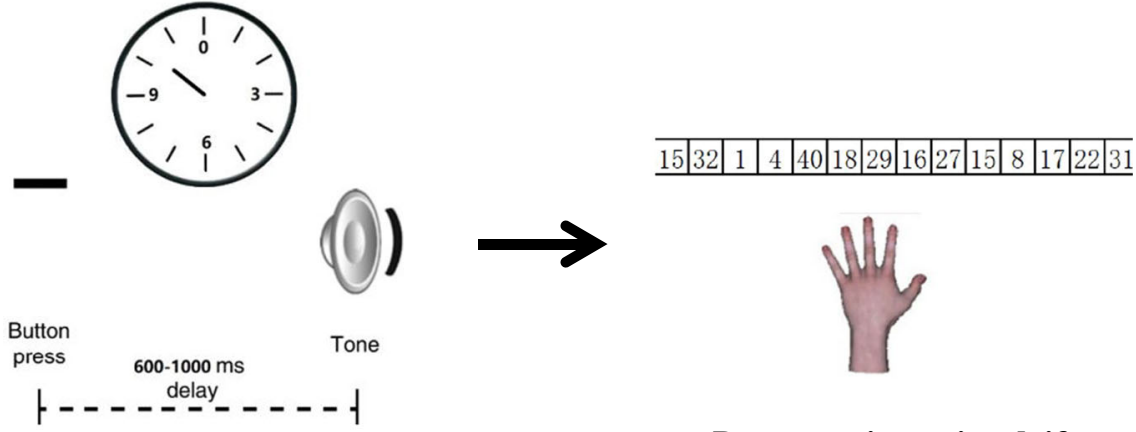

Baseline test, 10 IB task
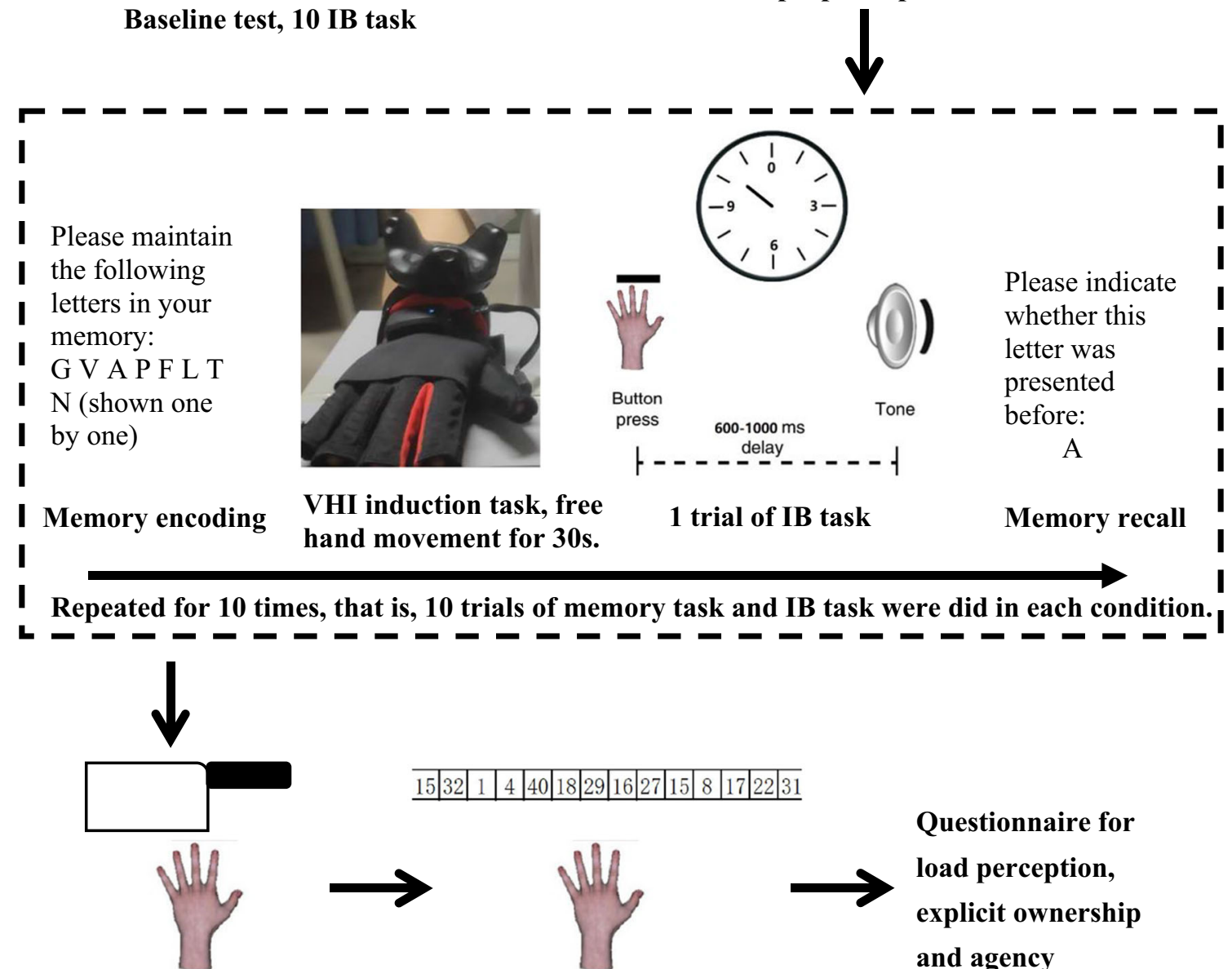

\section{Threat stimulus for SCR}

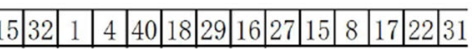
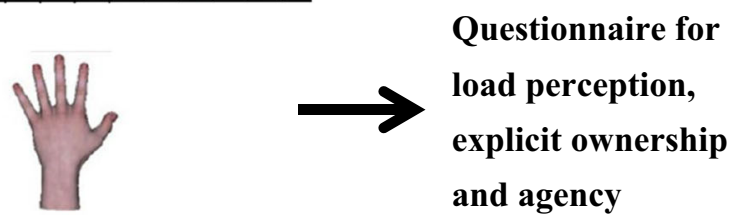

\section{Post-proprioceptive drift}

Fig. 2 Schematic overview of one specific condition example. Baseline test that contains ten intentional binding (IB) tasks was conducted before the actual experiment. Each experimental condition started with the pre-

(Ma \& Hommel, 2015b) were presented in the virtual environment, in which each number was set to be $1 \mathrm{~cm}$ wide (see Fig. 2). Participants were asked to put their real right hand on the fixed position on the real desk with the help of experimenter, ignoring the position of the virtual hand, but verbally report a number in the array to indicate the felt position of their real right middle finger. The sequence of numbers in the array was changed in each condition to prevent individual strategies. This was the pre-proprioceptive drift phase. proprioceptive drift, and ended with the questionnaire. There was a 2-min break between each two conditions. The experience sequences of the four conditions were counterbalanced across participants

The second phase consisted of ten trials, each of which contained one memory trial and one IB trial, as shown in Fig. 2. Participants were presented with a list of either two or eight randomly computer-generated letters, which they were required to hold in memory during the entire trial. After that, participants were asked to freely move their real right hand and watch the (synchronously or asynchronously moving) virtual hand movement for $30 \mathrm{~s}$. Then the virtual clock, pointer and button appeared again, and participants performed the same IB task as 
in the baseline IB test, with only one modification - the virtual hand was now shown and participants could control it (without or with delay for synchronous or asynchronous condition), and watch the contact between the virtual hand and the button. Participants again pressed the space key, waited for the tone to occur, and reported the pointer positions related to the tone. The tone was always triggered by the real contact, that is, for the IB task the only difference between synchronous and asynchronous conditions is the seen movement of the virtual hand. One second later after the pointer rotated back to zero, the virtual clock, its pointer, and the button disappeared again; the probe letter appeared on the screen and participants were to report whether or not it was presented during encoding (the recall stage). In total, the second phase contained ten trials, and each trial contained a trial of the memory task and the IB task, that is, participants reported ten positions for ten IB trials and recalled ten memory sets.

In the third, threat phase, participants were asked to place their real right hand in a relaxed state on the desk again, and watch a virtual knife appear, approach, and eventually cut the virtual hand, return to its original position, pause for $8 \mathrm{~s}$, and then cut again. The SCR was recorded continuously during the entire experiment (Ma \& Hommel, 2013). The virtual knife cut the virtual hand four times in each condition. Then participant underwent a post-proprioceptive phase to indicate their felt position of the middle finger of the unseen real hand, just as in the pre-proprioceptive phase.

Finally, participants were asked to fill in the agency, ownership, and a cognitive load questionnaire. There was a 2-min break after each condition, in order to reduce participant fatigue and prevent possible interference between the conditions.

\section{Questionnaire}

In line with an earlier study (Ma et al., 2019a; Ma \& Hommel, 2015b), we used an adapted Chinese version of the RHI/VHI questionnaire. We presented participants with ten questions assessing perceived agency (Q1-4), hand ownership (Q5-8), and the perceived cognitive load (Q9-10). For each statement, participants responded by choosing a score on a 7-point (1-7) Likert scale, for Q1-8, 1 indicating "strongly disagree," 4 indicating "uncertain," and 7 indicating "strongly agree," while for Q9-10, 1 indicated "very little," 4 indicated "moderate," and 7 indicated "very much." The statements were:

Q1. The movement of the virtual hand in the virtual environment was caused by me.

Q2. I can control the virtual hand.

Q3. The virtual hand on the screen followed my wishes.

Q4. When I make movements with my own hand, I expect the virtual hand to do the same movements with me.
Q5. I felt as if I was looking at my own hand when I was looking at this virtual hand.

Q6. I felt as if the virtual hand was my own hand.

Q7. I felt as if the virtual hand were a part of my body.

Q8. It seemed my right hand was at the same location as where the virtual hand was.

Q9. To what extent did you feel your cognitive load during the memory task?

Q10. How much cognitive effort did you spend during the memory task?

\section{Intentional binding (IB) task}

The IB task we used was the same as in our previous study (Ma et al., 2019b): a simplified Libert clock paradigm (Haggard et al., 2002) modeled after similar IB studies (Ma et al., 2019b; Ruess et al., 2018; Saito et al., 2015). Participants were to estimate the clock pointer position at the onset of the tone, as the timepoint of the keypress was always zero, so that the time interval between action and tone could be easily computed as the difference between the mean estimates in the experimental conditions and the baseline test (Ruess et al., 2018). Specifically, we first subtracted the perceived time interval reported by participants from the real time interval recorded with the script, and computed the ratio between this subtraction and the real time interval (Braun et al., 2014; Ma et al., 2019b). Next, we subtracted the baseline IB ratio from the ratio in each condition (Haggard et al., 2002). The expected compression of the perceived time interval (the IB effect) would correspond to a positive underestimated time interval, that is, more positive values correspond to more time compression (reduction), which in turn is assumed to reflect stronger agency (Ma et al., 2019b).

\section{Results}

From the available dependent measures, six variables were created. Responses to questionnaire items Q9 and Q10 were averaged to create a measure of perceived cognitive load, accuracy scores from the memory task served as recall measures, the average of Q5-8 as an explicit measure of perceived ownership, the average of Q1-4 as an explicit measure of perceived agency, the proprioceptive drift score as an implicit measure of ownership, and the IB as an implicit measure of agency. To derive SCR, the second implicit ownership measure, we defined a latency onset window between 1 and $8 \mathrm{~s}$ after the stimulus/event onset, namely when the virtual knife cut the virtual hand, with the skin conductivity before event onset serving as baseline (Ma \& Hommel, 2013, 2015b). We then calculated the magnitude of the event-induced SCR by subtracting baseline skin conductivity from the peak 

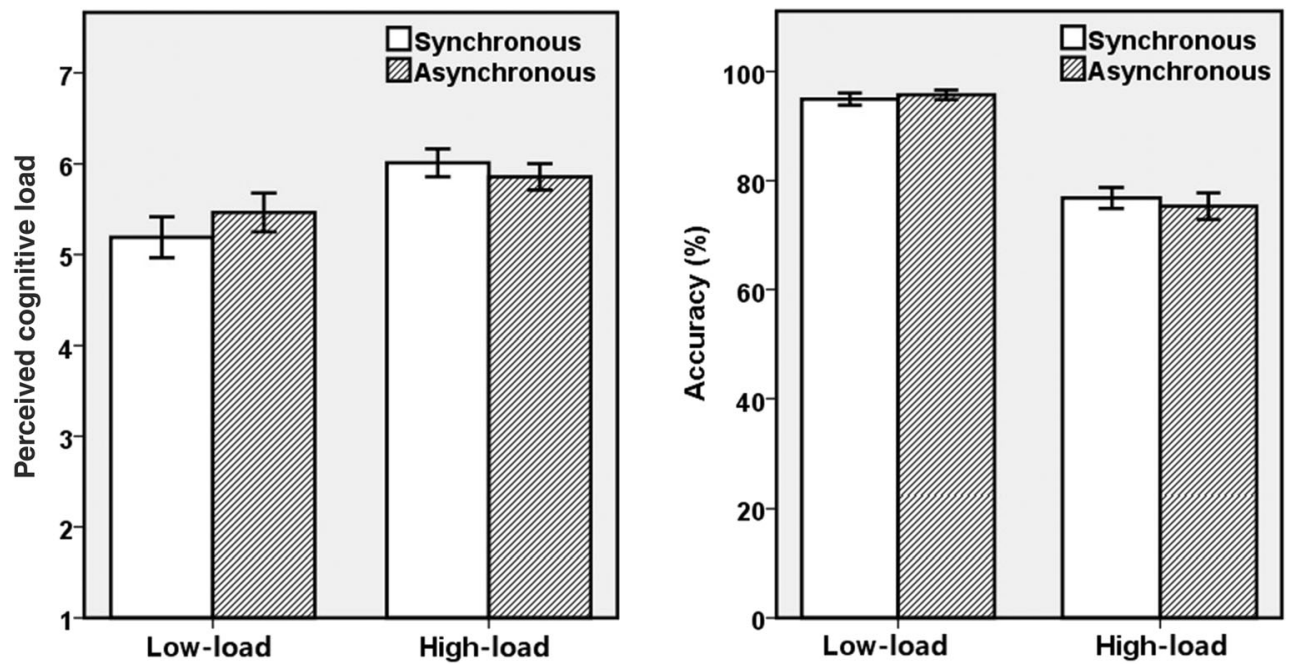

Fig. 3 Participants' (left panel) subjective ratings of cognitive load and (right panel) accuracy (\%) in the memory tasks as a function of load and synchrony. Error bars represent $\pm 1 \mathrm{SE}$

amplitude of the SCR during the analyzed time window, and took the $\log ($ magnitude +1$)$ per participant and condition (Figner \& Murphy, 2010). All six dependent variables were analyzed by means of a 2 (load) $\times 2$ (synchrony) repeatedmeasure ANOVA.

\section{Cognitive load (manipulation check)}

\section{Perceived cognitive load}

The ANOVA revealed a significant main effect of load, $\mathrm{F}(1,41)=14.55, \mathrm{p}<0.001, \mathrm{p}^{2}=0.26$, indicating that participants perceived lower cognitive load after finishing the low-load (mean $=5.33, \mathrm{SE}=0.20)$ than the high-load memory task (mean $=5.94, \mathrm{SE}=0.14)$. While the main effect of synchrony was not significant, $p>0.60$, the interaction was, $F(1,41)=$ $5.04, \mathrm{p}=0.03, \mathrm{p \eta}^{2}=0.11$. The load effect was more pronounced with synchrony, but two-tailed paired t-tests confirmed that it was significant in both synchrony, $\mathrm{t}(41)=4.26, \mathrm{p}<.001, \mathrm{~d}=0.67$, and asynchrony conditions, $\mathrm{t}(41)=2.20, \mathrm{p}=.033, \mathrm{~d}=0.34$. There was no significant synchrony effect in either low-load, $p=0.125$, or high-load conditions, $\mathrm{p}=0.190$.

\section{Recall}

In the ANOVA, only a significant main effect of load was found, $\mathrm{F}(1,41)=192.57, \mathrm{p}<0.001, \mathrm{p \eta}^{2}=0.82$, indicating that accuracy was lower under high $($ mean $=76.07, \mathrm{SE}=1.60)$ than under low cognitive load (mean $=95.32, \mathrm{SE}=0.84$ ). No other effect was significant, ps $>0.47$. This finding indicates that our cognitive load manipulation was successful (Fig. 3).

\section{Explicit measures}

\section{Ownership}

The ANOVA revealed a significant main effect of synchrony, $\mathrm{F}(1,41)=39.74, \mathrm{p}<0.001, \mathrm{p \eta}^{2}=0.49$, indicating that participants perceived stronger ownership over the virtual hand after having been exposed to the synchronous (mean $=4.21, \mathrm{SE}=$ 0.21 ) compared with the asynchronous condition (mean = $3.05, \mathrm{SE}=0.18)$. The load effect was not significant, $\mathrm{p}>$ 0.70 , but the interaction was, $\mathrm{F}(1,41)=5.79, \mathrm{p}=0.021$, $\mathrm{p \eta}^{2}=0.12$, showing that the synchrony effect was larger under the high-load than under the low-load condition. Two-tailed paired t-tests confirmed that the synchrony effect was significant in both low-load, $\mathrm{t}(41)=4.74, \mathrm{p}<.001, \mathrm{~d}=0.68$, and high-load conditions, $\mathrm{t}(41)=6.57, \mathrm{p}<.001, \mathrm{~d}=1.00$. There was no significant load effect in either synchrony, $p=0.117$, or asynchrony conditions, $\mathrm{p}=0.158$. See Fig. 4 .

\section{Agency}

The ANOVA revealed a significant main effect of synchrony, $\mathrm{F}(1,41)=59.29, \mathrm{p}<0.001, \mathrm{p \eta}^{2}=0.59$, indicating that participants perceived stronger agency over the virtual hand after having been exposed to synchronous (mean $=6.17, \mathrm{SE}=$ 0.10 ) compared with asynchronous conditions (mean $=4.44$, $\mathrm{SE}=0.21$ ). The main effect of load was not significant, $\mathrm{p}>$ 0.4 , but the interaction with synchrony was, $\mathrm{F}(1,41)=4.79$, $\mathrm{p}=0.034, \mathrm{p \eta}^{2}=0.11$, showing that the synchrony effect was larger under the high-load than under the low-load condition. Two-tailed paired $\mathrm{t}$-tests confirmed that the synchrony effect was significant in both low-load, $\mathrm{t}(41)=6.11, \mathrm{p}<.001, \mathrm{~d}=$ 1.18 , and high-load conditions, $\mathrm{t}(41)=8.36, \mathrm{p}<.001, \mathrm{~d}=$ 

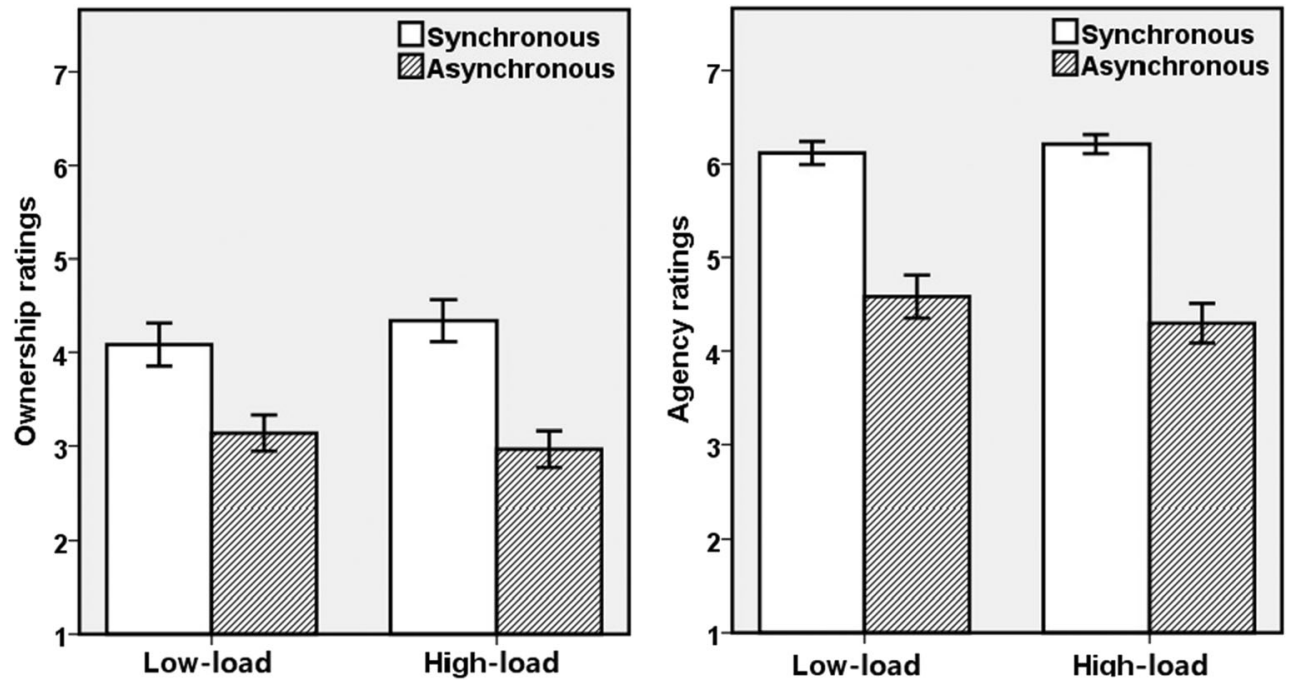

Fig. 4 (Left panel) Aggregated ownership and (right panel) agency question ratings as a function of load and synchrony. Error bars represent \pm 1 SE

1.61. There was no significant load effect in either synchrony, $p=0.431$, or asynchrony conditions, $p=0.093$. See Fig. 4 .

Given the strong similarity between the findings for ownership and agency judgments, we ran a MANOVA on ownership and agency scores with load, synchrony, and variable (ownership, agency) as independent variables. Importantly, the interaction between cognitive load and synchrony was again significant $\left(\mathrm{F}(1,41)=8.66, \mathrm{p}=0.005, \mathrm{p \eta}^{2}=0.17\right)$, while the three-way interaction was not ( $p=0.83)$, suggesting that explicit ownership and agency were equally affected by the load-synchrony interaction.

\section{Implicit measures}

\section{Proprioceptive drift}

The ANOVA yielded significant main effects of cognitive load, $\mathrm{F}(1,41)=5.94, \mathrm{p}=0.019, \mathrm{p \eta}^{2}=0.13$, and synchrony, $\mathrm{F}(1,41)=7.23, \mathrm{p}=0.01, \mathrm{p}^{2}=0.15$, indicating that drift rates were more pronounced and more positive under low load $($ mean $=1.08, \mathrm{SE}=0.38)$ than under high load (mean = $0.31, \mathrm{SE}=0.40$ ); and with synchrony (mean $=1.04, \mathrm{SE}=$ 0.34 ) than with asynchrony (mean $=-0.26, \mathrm{SE}=0.37$ ). The interaction effect was far from significant, $\mathrm{p}>0.7$. See Fig. 5.

\section{Skin conductance response (SCR)}

The ANOVA revealed a significant main effect of cognitive load, $\mathrm{F}(1,41)=4.45, \mathrm{p}=0.041, \mathrm{p \eta}^{2}=0.10$, indicating that $\mathrm{SCR}$ was higher under low load (mean $=0.104, \mathrm{SE}=0.02$ ) than under high load (mean $=0.085, \mathrm{SE}=0.02$ ). No other effect was significant, ps $>0.5$. See Fig. 5 .

\section{Intentional binding}

The ANOVA revealed a significant main effect of cognitive load, $\mathrm{F}(1,41)=4.96, \mathrm{p}=0.031, \mathrm{p \eta}^{2}=0.11$, indicating more pronounced IB (time compression) under low load (mean = 4.74, $\mathrm{SE}=0.88)$ than under high load (mean $=2.92, \mathrm{SE}=$ 1.03). No other effect was significant, $p s>0.9$. Separate tests (with Bonferroni correction for multiple comparisons; $\mathrm{p} \leq$ 0.0125 ) showed that the IB effect was significant in the lowload-synchronous condition, $\mathrm{t}(41)=5.29, \mathrm{p}<0.001, \mathrm{~d}=0.82$, in the low-load-asynchronous condition, $\mathrm{t}(41)=4.06, \mathrm{p}<$ $0.001, \mathrm{~d}=0.63$, and in the high-load-synchronous condition, $\mathrm{t}(41)=2.70, \mathrm{p}=0.010, \mathrm{~d}=0.42$; but only close to significance in the low-load-synchronous condition, $\mathrm{t}(41)=2.60, \mathrm{p}=$ $0.013, d=0.40$. See Fig. 6 .

\section{Post hoc power analysis}

Effect sizes $\left(\mathrm{p}^{2}\right)$ for load or synchrony manipulations in all measures, including questionnaire results, proprioceptive drift, SCR, and IB, were higher than 0.10 for main effects and interactions. A power analysis using G*Power 3 (Faul et al., 2009), with $\alpha=0.05$, showed that power (1- $\beta$ ) was higher than 0.99 , suggesting that the sample size was sufficient for us to detect the sought-for differences.

\section{Discussion}

The major aim of the present study was to investigate the impact of cognitive load on explicit and implicit indicators of body ownership and agency. The outcome is very clear and systematic in showing comparable patterns for the two explicit measures - no main effect of cognitive load but an 


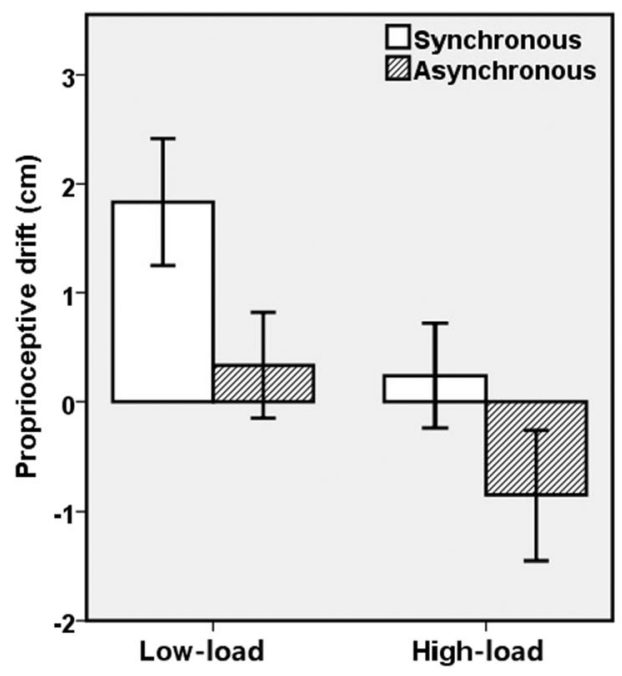

Fig. 5 Results for (left panel) proprioceptive drift; (right panel) and for skin conductance response (SCR) as a function of load and synchrony. A positive drift value indicates that the felt position of the real hand was

interaction between load and synchrony - and comparable patterns for the three implicit measures - load main effects but no interaction. Hence, the load manipulation structured the measures according to their explicit versus implicit nature but not with respect to ownership or agency. Interestingly, the direction of the effects including load was opposite for explicit and implicit measures. The interactions obtained for the explicit measures indicate that higher load, presumably by diminishing top-down contributions to the respective judgment (Hon et al., 2013), increased the impact of synchrony, an assumed strong bottom-up source for ownership judgments (Botvinick \& Cohen, 1998). In contrast, the main effects of

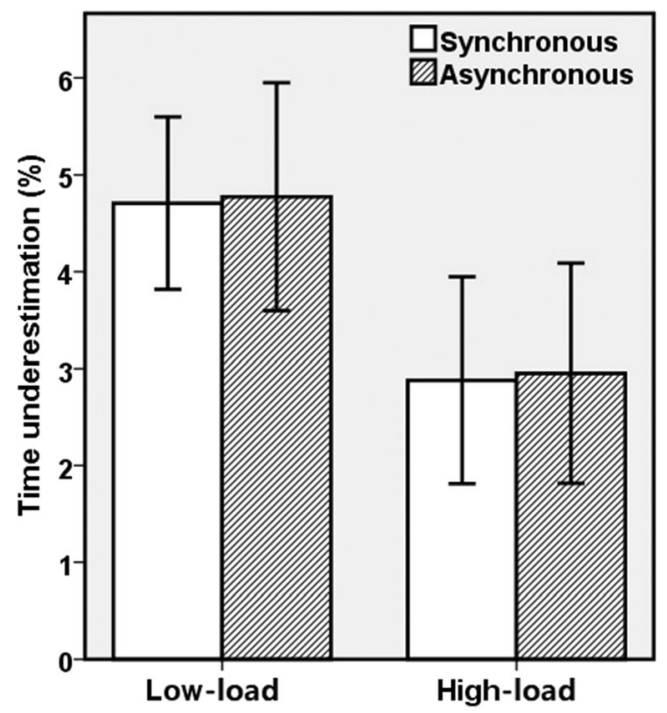

Fig. 6 Mean underestimation of time interval as a function of load and synchrony. Higher ratio (\%) indicates more underestimation, and thus stronger IB. Error bars represent $\pm 1 \mathrm{SE}$

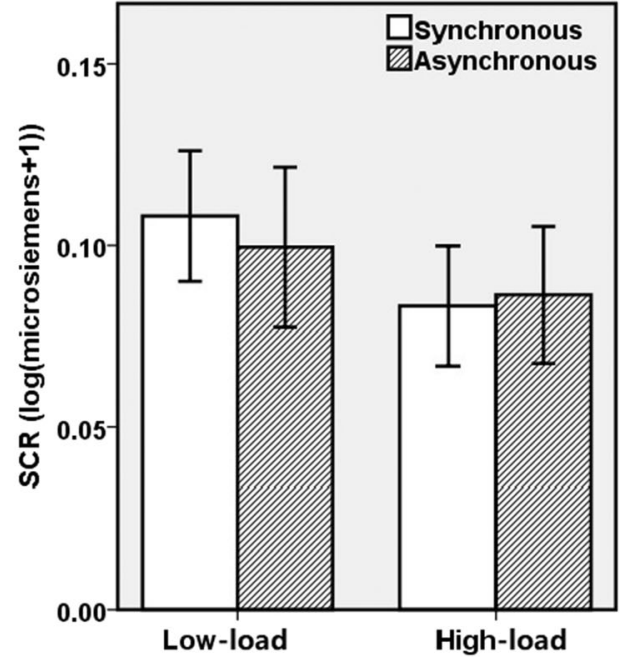

shifted to the virtual hand; a higher SCR indicates stronger arousal accompanied threatening of the virtual hand. Error bars represent $\pm 1 \mathrm{SE}$

load for the implicit measures indicate that higher load impaired the processing of information underlying the respective effect. This provides clear evidence that explicit and implicit measures rely on different informational sources that are differentially affected by the load manipulation.

The manipulation check of cognitive load contained explicit subjective ratings and accuracy percentages. While the accuracy percentages were similar to previous findings (Howard et al., 2016), thus confirming that the manipulation worked, the explicit subjective ratings, a new measure, indicated a rather weak effect of load. While the load effect was significant in both synchrony conditions, the ratings showed only rather small numerical differences and they were all higher than five, thus indicating that perceived load was rather high in all conditions. We speculate that this might reflect our experimental procedure: whereas accuracy was computed per trial, subjective ratings were recorded for one specific condition, containing ten trials, which might have increased the perception of difficulty.

To better understand the discrepancy between the result patterns obtained for explicit and implicit measures, it is important to relate the type of information needed to generate the phenomena being measured to the cognitive process targeted by our load manipulation. Note that we used a workingmemory task to induce different loads, as such tasks are known to interfere with cognitive-control processes and the regulation of top-down control (Diamond, 2013; Miyake \& Shah, 1999). The character of this task renders it likely that it interferes with the short-term maintenance of information and the comparison of information obtained and different points in time. Ownership accounts that allow for multiple informational contributions commonly assume that overlearned, 
generalized knowledge about one's own body is used to bias and select incoming sensory information that is relevant for experiencing one's body (e.g., Apps \& Tsakiris, 2014; Synofzik et al., 2008a; Tsakiris, 2017). Agency accounts assume that agency judgments are derived from the degree to which predicted sensory effects of an intentional action match the actual state (Frith et al., 2000; Moore \& Haggard, 2008) that is, the degree to which intended effects have actually been achieved. It is important to consider that the effect of synchrony actually rests on an illusion that would not exist if ownership and agency judgments would only rely on the top-down contributions to the perception of ownership and agency, that is, on the overlearned expectations regarding what one's body looks like and what sensory effects one's actions should have. This means that top-down contributions should reduce the illusion, which fits our present observation that impairing top-down contributions leads to an increase of the illusion, that is, to more pronounced effects of synchrony on perceived ownership and agency.

This scenario is different for implicit measures. For explicit measures, the working-memory manipulation was likely to affect the top-down component only but not the online bottom-up contributions from sensory sources. For implicit measures, however, the load manipulation is likely to have interfered with the collection of the information represented by the measures itself. For all three implicit measures used in the present study, the obtained effect relies on the retrieval or maintenance of information over time, that is, on the use and/ or comparison of information obtained and processed at different points in time.

This is obvious for the drift measure. The drift is calculated by relating the degree to which localizing one's real hand is affected by the location of the virtual hand before and after a particular synchrony manipulation. If these two measures differ, this must be because the synchrony manipulation in the immediately preceding experimental phase has changed the difficulty of ignoring the virtual hand. If one's memories of the synchrony manipulation and/or the resulting change in representing the virtual hand were flushed, the drift should be zero. On the one hand, this is clearly not what happened in our study, because a main effect of synchrony was obtained for drift rates. On the other hand, however, it is interesting to note that the high-load condition did not generate any positive drift: drift was close to, and statistically not different from, zero with synchrony and negative with asynchrony. Even though we have no explanation to offer for this particular pattern, it seems clear that the typical positive drift rates could not be obtained in the high-load condition, suggesting that impairing working memory interfered with the processes responsible for positive drift rates.

A role for memory maintenance processes is also obvious with respect to the IB measure. The IB effect is based on a comparison between reports of memorized clock positions.
The working-memory manipulation must have impaired the maintenance of the information reflected by these reports, so that a smaller IB effect in the high-load condition makes perfect sense. The case is a bit different for SCR. The measure itself is likely to reflect current, ongoing internal states that are likely to do with emotion, rather than memory. However, these emotions do rely on interpretations of the observed situation as dangerous and threatening. These interpretations do rely on the retrieval of information from memory, and it makes sense to assume that this retrieval was more impaired under high load. Along the lines of this reasoning, we may thus conclude that load manipulations related to working memory are likely to increase effects that are dampened by top-down factors, like generalized expectations or predictions, and to reduce effects that rely on stored information, or integrate or compare information over time.

It may be interesting to note that the present IB effects were numerically lower than in previous studies, where the time underestimation was usually higher than $10 \%$ (Braun et al., 2014; Experiments 1 and 2 in Ma et al., 2019b; Ma et al., 2021). Even though the IB effect was significant or at least almost significant in all conditions, one may wonder why our effects were so small numerically. We speculate that the longer practice trials and the memory tasks have made the overall task rather challenging and demanding (as the subjective ratings indicate), which may have reduced the IB effect. This is consistent with an observation in a previous study of ours (Ma et al., 2019b): in Experiments 1 and 2 of this study, the VHIinducing manipulation was rather short ( $2 \mathrm{~min}$ ), and the time underestimation was around 10\%, while in Experiments 3 and 4 , the VHI-inducing manipulation was doubled in length (4 $\mathrm{min}$ ), and the time underestimation was substantially smaller. Combining this observation with our present findings suggests that higher cognitive load (due to longer task duration or dual-tasking) might reduce IB.

Finally, it is interesting to note that explicit ownership and agency were equally affected by our manipulations. Classical RHI studies (e.g., Kalckert \& Ehrsson, 2012) have commonly found discrepancies between perceived ownership and agency, which has been taken to support the theoretical claim that ownership and agency represent two different and separable components of the human minimal self (Gallagher, 2000). In contrast, studies using the ecologically more plausible VHI have provided evidence for a strong overlap and strong correlations between ownership and agency judgments (Ma \& Hommel, 2015b). The present study provides further support for a tight interrelation between perceived ownership and agency, which fits with previous observations from both our own lab and other research groups (Braun et al., 2018; Pyasik et al., 2018; Tsakiris et al., 2007). These findings raise the interesting theoretical possibility that both kinds of judgments are derived from strongly overlapping informational sources and that they integrate bottom-up and top-down contributions 
in comparable ways. If so, theories of agency might be extendable to account for ownership, and vice versa. For example, Ma et al. (2019a) provided evidence that theoretical principles proposed to account for agency (Wegner, 2003) also account for ownership -suggesting that Wegner's agency theory might provide a basis for an integrative framework of ownership and agency illusions.

Acknowledgements This research was supported by Chongqing Social Sciences Planning Project (2018BS96); National Natural Science Foundation of China (31700942); the Fundamental Research Funds for the Central Universities (SWU2009429) to K. M. in China, and a grant of the German Research Council (DFG: HO 1430/12-1) to B. H.

Open practices statement Raw data of the study are available on the Open Science Framework (https://osf.io/g85kd/), and the experiment was not preregistered.

Author contributions J. Q. and K. M. developed the study concept. All authors contributed to the study design. Testing and data collection were performed by J. Q. Data analysis and manuscript drafting were performed by J. Q. and K. M., and B. H. provided critical revisions. All authors approved the final version of the manuscript for submission.

Declarations The authors declare that they have no conflicts of interest with respect to their authorship or the publication of this article. All procedures performed in this study were in accordance with the ethical standards of the ethics committee in Southwest University and with the 1964 Helsinki Declaration and its later amendments. Informed consent was obtained from all participants included in this study.

\section{References}

Apps, M. A. J., \& Tsakiris, M. (2014). The free-energy self: A predictive coding account of self-recognition. Neuroscience \& Biobehavioral Reviews, 41, 85-97.

Armel, K. C., \& Ramachandran, V. S. (2003). Projecting sensations to external objects: Evidence from skin conductance response. Proceedings of the Royal Society of London, Series B: Biological Sciences, 270(1523), 1499-1506.

Blanke, O., \& Metzinger, T. (2009). Full-body illusions and minimal phenomenal selfhood. Trends in Cognitive Sciences, 13, 7-13. https://doi.org/10.1016/j.tics.2008.10.003

Botvinick, M., \& Cohen, J. (1998). Rubber hands' feel' touch that eyes see. Nature, $391,756$.

Braun, N., Thorne, J. D., Hildebrandt, H., \& Debener, S. (2014). Interplay of agency and ownership: the intentional binding and rubber hand illusion paradigm combined. PLOS ONE, 9: e111967.

Braun, N., Debener, S., Spychala, N., Bongartz, E., Sörös, P., Müller, H. H. O., \& Philipsen, A. (2018). The Senses of Agency and Ownership: A Review. Frontiers in Psychology, 9: 535.

Dewey, J. A., \& Knoblich, G. (2014). Do Implicit and Explicit Measures of the Sense of Agency Measure the Same Thing. PLoS ONE, 9(10): e110118.

Diamond, A. (2013). Executive functions. Annual Review of Psychology, 64, 135-168.

Ehrsson, H. H. (2012). "The concept of body ownership and its relation to multisensory integration," in The New Handbook of Multisensory Processes, ed. B. E. Stein (MIT Press), 775-792.
Ebert, J. P., \& Wegner, D. M. (2010). Time warp: Authorship shapes the perceived timing of actions and events. Consciousness and Cognition, 19(1), 481-489.

Faul, F., Erdfelder, E., Buchner, A., \& Lang, A. G. (2009). Statistical power analyses using $\mathrm{G}^{*}$ Power 3.1: Tests for correlation and regression analyses. Behavior Research Methods, 41(4), 1149-1160.

Figner, B., and Murphy, R. O. (2010). "Using skin conductance in judgment and decision making research," in A Handbook of Process Tracing Methods for Decision Research: A Critical Review and User's Guide, eds M. SchulteMecklenbeck, A. Küehberger, and R. Ranyard (Psychology Press), 163-184

Frith, C. D., Blakemore, S. J., \& Wolpert, D. M. (2000). Abnormalities in the awareness and control of action. Philosophical Transactions of the Royal Society B, 355(1404), 1771-1788.

Gallagher, S. (2000). Philosophical conceptions of the self: implications for cognitive science. Trends in Cognitive Sciences, 4(1), 14-21.

Gallagher, S. (2007). The natural philosophy of agency. Philosophy Compass, 2(2): 347-357

Haggard, P., Clark, S., \& Kalogeras, J. (2002). Voluntary action and conscious awareness. Nature Neuroscience, 5(4), 382-385.

Haggard, P. (2017). Sense of agency in the human brain. Nature Reviews Neuroscience, 18(4), 196-207.

Hon, N., Poh, J.-H., \& Soon, C.-S. (2013). Preoccupied minds feel less control: sense of agency is modulated by cognitive load. Consciousness and Cognition, 22(2), 556-561.

Howard, E. E., Edwards, S. G., \& Bayliss, A. P. (2016). Physical and mental effort disrupts the implicit sense of agency. Cognition, 157, 114-125.

Jeannerod, M. (2003). The mechanism of self-recognition in humans. Behavioural Brain Research, 142, 1-15.

Kalckert, A., \& Ehrsson, H. H. (2012). Moving a rubber hand that feels like your own: A dissociation of ownership and agency. Frontiers in Human Neuroscience, 6: 40.

Kannape, O. A., Barre, A., Aminian, K., \& Blanke, O. (2014). Cognitive Loading Affects Motor Awareness and Movement Kinematics but Not Locomotor Trajectories during Goal-Directed Walking in a Virtual Reality Environment. PLoS One, 9(1): e85560.

Ma, K., \& Hommel, B. (2013). The virtual-hand illusion: Effects of impact and threat on perceived ownership and affective resonance. Frontiers in Psychology, 4: 604.

Ma, K., \& Hommel, B. (2015a). Body-ownership for actively operated non-corporeal objects. Consciousness and Cognition, 36, 75-86.

Ma, K., \& Hommel, B. (2015b). The role of agency for perceived ownership in the virtual hand illusion. Consciousness and Cognition, 36, 277-288.

Ma, K., Hommel, B., \& Chen, H. (2019a). The roles of consistency and exclusivity in perceiving body ownership and agency. Psychological Research, 83, 175-184.

Ma, K., Hommel, B., \& Chen, H. (2019b). Context-induced contrast and assimilation in explicit and implicit measures of agency. Scientific Reports, 9: 3883.

Ma, K., \& Hommel, B. (2020). Metacontrol and body ownership: divergent thinking increases the virtual hand illusion. Psychological Research, 84(1), 120-127.

Ma, K., Qu, J., Yang, L., Zhao, W., \& Hommel, B. (2021). Explicit and implicit measures of body ownership and agency: affected by the same manipulations and yet independent. Experimental Brain Research.

Miyake, A., \& Shah, P. (1999). Toward unified theories of working memory: Emerging general consensus, unresolved theoretical issues, and future research directions. In A. Miyake \& P. Shah (Eds.), Models of working memory: Mechanisms of active maintenance and executive control (pp. 442-481). Cambridge Univ. Press.

Moore, J., \& Haggard, P. (2008). Awareness of action: Inference and prediction. Consciousness and Cognition, 17(1), 136-144. 
Moore, J. W., and Fletcher, P. C. (2012). Sense of agency in health and disease: a review of cue integration approaches. Consciousness and Cognition. 21, 59-68. https://doi.org/10.1016/j.concog.2011.08.010

Pfister, R., Klaffehn, A. L., Kalckert, A., Kunde, W., \& Dignath, D. (2020). How to lose a hand: sensory updating drives disembodiment. Psychonomic Bulletin \& Review. https://doi.org/ 10.3758/s13423-020-01854-0

Pyasik, M., Burin, D., \& Pia, L. (2018). On the relation between body ownership and sense of agency: A link at the level of sensory-related signals. Acta Psychologica, 185, 219-228.

Pyasik, M., Tieri, G., \& Pia, L. (2020). Visual appearance of the virtual hand affects embodiment in the virtual hand illusion. Scientific Reports, 10: 5412.

Ruess, M., Thomaschke, R., \& Kiesel, A. (2018). Intentional binding of visual effects. Attention Perception \& Psychophysics, 80(3), 713722

Saito, N., Takahata, K., Murai, T., \& Takahashi, H. (2015). Discrepancy between explicit judgement of agency and implicit feeling of agency: Implications for sense of agency and its disorders. Consciousness and Cognition, 37, 1-7.

Sanchez-Vives, M. V., Spanlang, B., Frisoli, A., Bergamasco, M., \& Slater, M. (2010). Virtual hand illusion induced by visuomotor correlations. PLOS ONE, 5: e10381.

Slater, M., Perez-Marcos, D., Ehrsson, H. H., \& Sanchez-Vives, M. V. (2008). Towards a digital body: The virtual arm illusion. Frontiers in Human Neuroscience, 2: 6.
Sternberg, S. (1966). High-speed scanning in human memory. Science, 153(3736), 652-654.

Synofzik, M., Vosgerau, G., \& Newen, A. (2008a). Beyond the comparator model: a multifactorial two-step account of agency. Consciousness \& Cognition, 17, 219-239.

Synofzik, M., Vosgerau, G., and Newen, A. (2008b). I move, therefore I am: a new theoretical framework to investigate agency and ownership. Consciousness and Cognition, 17, 411-424.

Tsakiris, M., \& Haggard, P. (2005). The rubber hand illusion revisited: Visuotactile integration and self-attribution. Journal of Experimental Psychology: Human Perception and Performance, $31,80-91$.

Tsakiris, M., Schütz-Bosbach, S., \& Gallagher, S. (2007). On agency and body-ownership: Phenomenological and neurocognitive reflections. Consciousness and Cognition, 16, 645-660.

Tsakiris, M. (2017). The multisensory basis of the self: From body to identity to others. Quarterly Journal of Experimental Psychology, $70(4), 597-609$.

Wegner, D. M. (2003) The mind's best trick: how we experience conscious will. Trends in Cognitive Sciences, 7, 65-69.

Publisher's note Springer Nature remains neutral with regard to jurisdictional claims in published maps and institutional affiliations. 